\title{
Cerebrospinal fluid leakage and pneumocephalus secondary to spine stab wounds
}

\author{
Nimrod Rahamimov $\cdot$ Hani Mulla $\cdot$ \\ Shay Freiman
}

Received: 19 June 2009/Accepted: 14 September 2009/Published online: 14 November 2009

(C) Springer-Verlag 2009

\begin{abstract}
Cerebro-spinal fluid leakage from a spinal stab wound is rare, and usually not a life-threatening injury. Pneumocephalus, a possible rare complication of traumatic cerebro-spinal fluid leakage, can potentially lead to intracranial hemorrhage and death. We describe two cases of spinal stab wounds complicated by cerebro-spinal fluid leakage, one of them developing into severe pneumocephalus. Both patients recovered completely with the following treatment protocol: (a) trendelenburg positioning of the patient, (b) insertion of a continuous-drainage cerebro-spinal fluid catheter into the lumbar thecal sac, (c) primary suture of the leaking skin wound, (d) IV antibiotic therapy.
\end{abstract}

Keywords Spine - Pneumocephalus - Pneumorrhachis · Stab wound $\cdot$ CSF

\section{Introduction}

External cerebrospinal fluid (CSF) leakage from a stab wound to the spine is a rare occurrence. Peacock et al. [1], in a review of 450 patients with stab wounds of the spinal cord, found only 18 (4\%) to have an external skin CSF leak. They note that in the majority of these patients the leakage stopped spontaneously, and no surgical intervention was necessary.

This corresponds well with the general agreement in the literature that surgical treatment of penetrating spine

N. Rahamimov $(\square) \cdot$ H. Mulla $\cdot$ S. Freiman Spine Surgery Unit, Western Galilee Hospital, P.O. Box 21, Naharia 22100, Israel

e-mail: nimrod.rahamimov@naharia.health.gov.il; nim205@gmail.com injuries is usually unwarranted if there is no retained foreign body, sepsis, or CSF leakage lasting more than 3 4 days [2].

Neurological injury is typically a Brown-Sequard lesion, which has a relatively good prognosis for spontaneous recovery. Other cord or root injuries will usually not improve much with or without surgery, so neurological loss per se is not an indication for surgical treatment either $[1,3]$.

One potentially life-threatening complication of CSF leakage is pneumocephalus. Entrance of air into the cranial cavity is thought to be due to one of two possible mechanisms [3, 4]: a drop in intracranial pressure (ICP), causing a vacuum effect which results in an influx of air with respiration and coughing; or a valve effect at the wound site that allows air to enter the subarachnoid space but not to exit.

We describe two cases of CSF leakage following stab wounds to the spine, one of them developing into severe pneumocephalus.

\section{Case reports}

Both patients gave their consent to the publication of their clinical histories.

Case 1

A 32-year old female was admitted to the Chest Surgery Unit following a domestic altercation in which she sustained five stab wounds to the posterior thorax, with a resultant small hemopneumothorax. The skin wounds were superficially sutured. A chest drain was not inserted, and the patient was hospitalized for observation. 


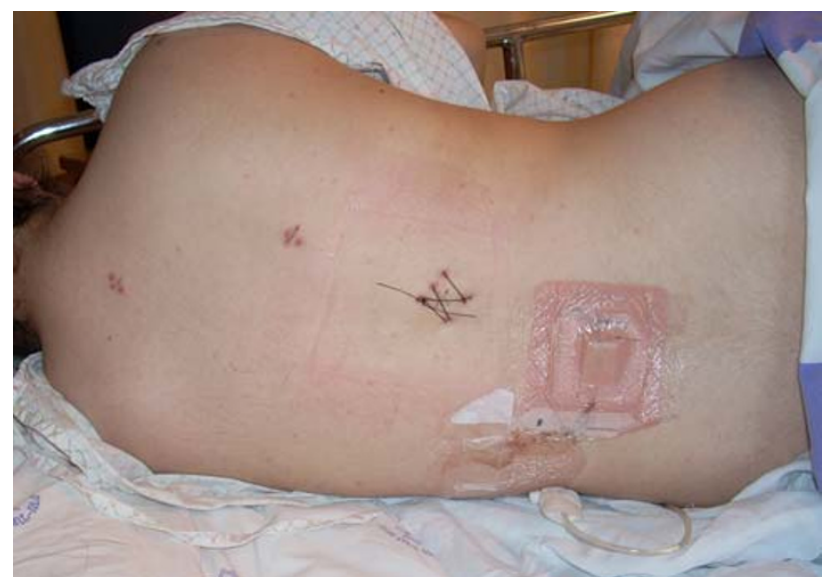

Fig. 1 Re-sutured stab wound and continuous drainage catheter

On admission, the patient's chief complaint was headaches. No meningeal irritation or neurological deficit was noted. On the fourth day of hospitalization, one of the stab wounds, at the T9-10 level, was found to be leaking clear fluid suspected to be CSF.

An intradural continuous drainage (CD) 16G catheter was inserted into the lumbar spine at the L4-5 level, and the leaking skin wound was re-sutured with through and through sutures (Fig. 1). The patient was remanded to bed in a supine Trendelenburg position with immediate resolution of the headaches, and intravenous (IV) antibiotics were initiated.).

After five days, with the stab wound healing with no sign of leak or infection, the CD catheter was removed and

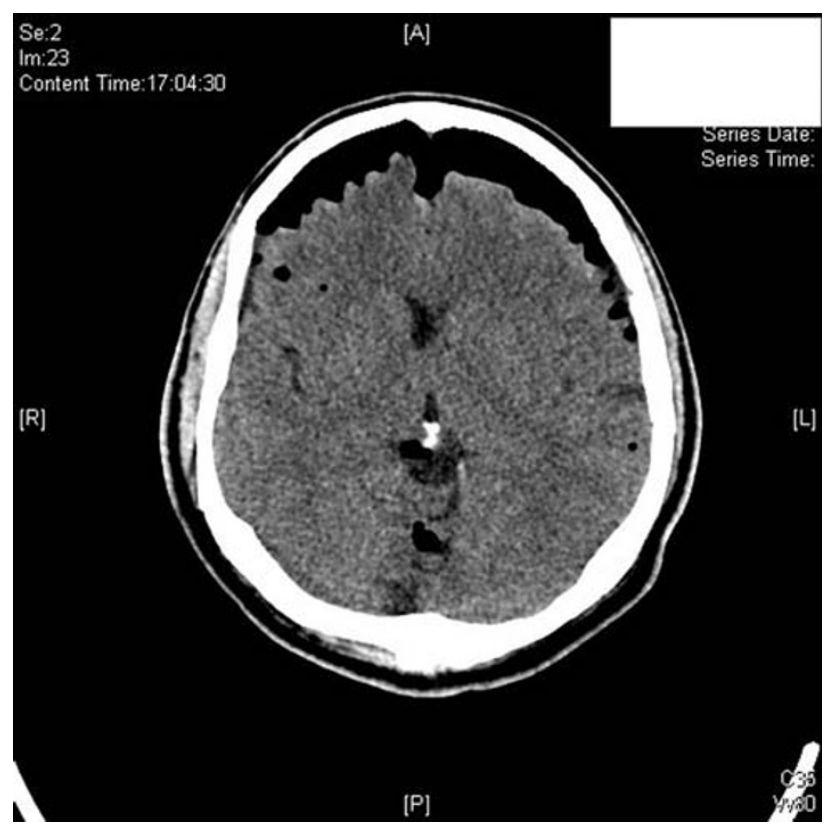

Fig. 2 Severe pneumocephalus

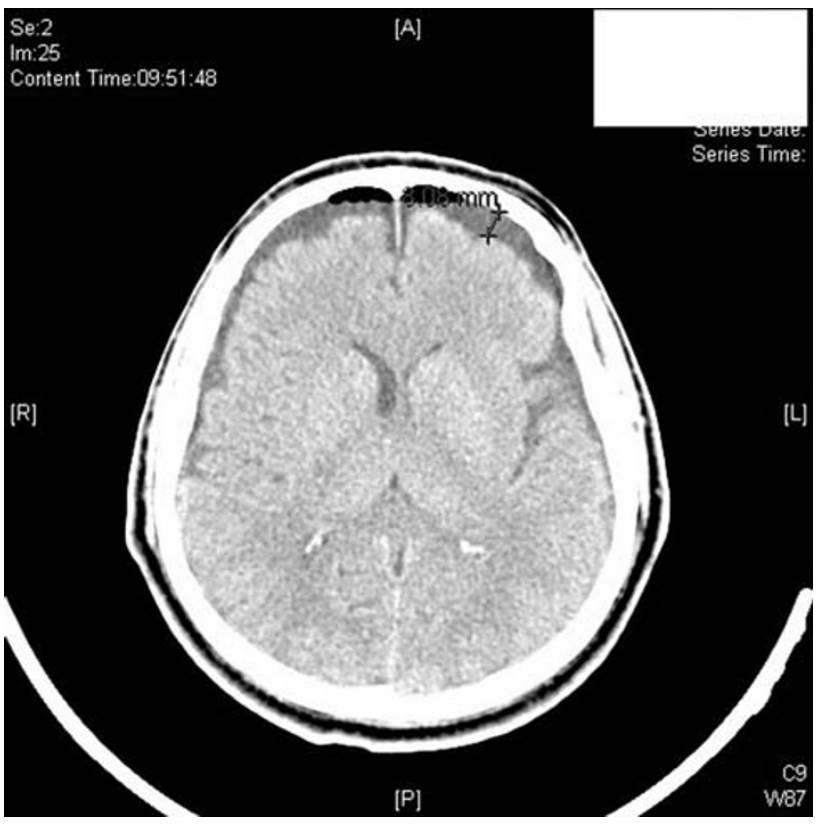

Fig. 3 Resolving pneumocephalus following treatment described

the patient was allowed to ambulate. She was discharged with no sequela on follow-up.

Case 2

A 66-year old male in mild shock was brought to the ER with two stab wounds to the lower back and posterior thigh, sustained in the process of resolving a criminal dispute.

On admission, the patient was found to be fully conscious, with a Glasgow Coma Score of 15, blood pressure 90/62, pulse 82 (Revised Trauma Score 12). Arterial bleeding from the thigh wound was noted, originating from a laceration of the profunda femoris artery, which was embolized. Three units of packed cells were given, with the hemoglobin concentration rising from 7.2 to $9.5 \mathrm{mg} \%$.

The patient was admitted to the respiratory intensive care unit, where he was hemodynamically stable but complained of severe headaches. On the next day, the dressing over the lower back stab wound was noted to be drenched in clear fluid presumed to be CSF. A brain computed tomography (CT) scan demonstrated severe pneumocephalus (Fig. 2). An attempt was made to explore and directly suture the dural tear, but this failed.

A CD catheter was inserted into the lumbar spine at the L4-5 level, and the skin wound was sutured with through and through sutures. The patient was placed in a supine Trendelenburg position. The headaches resolved gradually over a three-day period, with follow-up CT demonstrating a significant reduction in the subarachnoid air volume (Fig. 3). After five days, with the stab wound dry and 


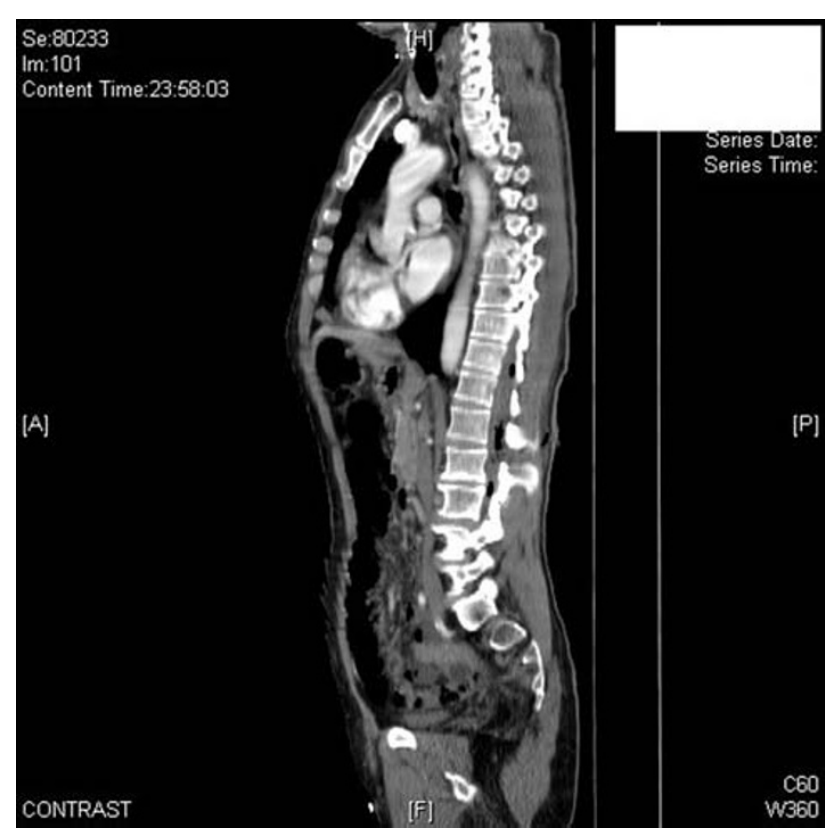

Fig. 4 Air bubbles in spinal canal near skin wound site

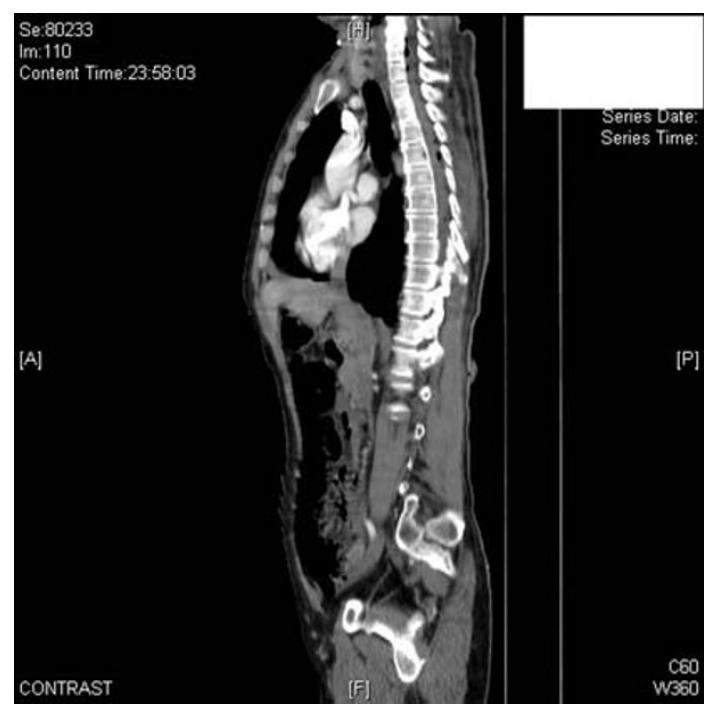

Fig. 5 Air bubbles ascending the spinal canal

closed, the CD catheter was removed and the patient began to ambulate. He was discharged from the hospital with no sequela.

\section{Discussion}

In both of the cases we have described here, the CSF leakage responded well to nonsurgical measures. Insertion of a $\mathrm{CD}$ catheter reduces the intradural pressure and consequently decreases the CSF outflow through the traumatic dural laceration, allowing it to close and heal.
Placing the patient in the Trendelenburg position accomplishes two objectives. The first is to reduce the hydrostatic pressure within the dural sac at the dural laceration site, and the second is to prevent pneumocephalus by having the head lower than the possible air-entry site, thereby making the air bubbles ascend the raised caudal thecal sac instead of passing into the cranial cavity.

Primary closure of the contaminated skin wound increases the risk of infection, but this consideration is outweighed in our opinion by the importance of preventing air from being withdrawn into the wound.

Pneumocephalus develops in about $78 \%$ of patients with traumatic pneumorrhachis (air in the spinal canal) [5]. In our second case, air bubbles were clearly seen in the initial CT scan done on arrival (Figs. 4, 5). In a trauma setting, the finding of trapped intrathecal air in the spine should alert the clinician to search for a CSF leak and pneumocephalus. Even if no other measure is taken, simple tilting of the bed to a Trendelenburg position will stop the leak from becoming a potential neurosurgical emergency.

Antibiotic therapy should be directed at the prime contaminator of spine wounds-Staphylococcus aureus. In our hospital, methicillin resistant Staphylococcus aureus (MRSA) is the pathogen in $16 \%$ of all staphylococcal infections, so we prefer vancomycin for at-risk nosocomial infection prophylaxis.

Headaches are a common complaint after accidental durotomy during epidural injections and spinal surgery. Both of our patients complained of severe headaches that resolved when the CSF leak was treated.

In summary, in our experience, penetrating spine stab wounds with CSF leakage can be safely and effectively treated with a combination of continuous CSF drainage, Trendelenburg positioning, primary closure of the leaking skin wound, and IV antibiotic therapy.

Conflict of interest statement The authors declare that they have no conflict of interest related to the publication of this manuscript.

\section{References}

1. Peacock WJ, Shrosbree RD, Key AG (1977) A review of 450 stabwounds of the spinal cord. S Afr Med J 51:961-964

2. Page RD, Lye RH (1989) Stab wound of the neck: potential pitfalls in management. Arch Emerg Med 6:225-229

3. Uppot RN, Vinay GK, Gould SW, Hirohiko I (1999) Pneumocephalus and Brown-Sequard's neurologic injury caused by a stab wound to the neck. AJR 173:1504

4. Bunc G, Roskar Z, Vorsic M (2001) Pneumocephalus secondary to a neck stab wound without neurologic injury in a 13 year old girl. Pediatr Neurosurg 34:239-241

5. Goh BK, Yeo AW (2005) Traumatic pneumorrhachis. J Trauma 58:875-879 